\title{
Trends in microbiological spectrum of endophthalmitis at a single tertiary care ophthalmic hospital in India: a review of 25 years
}

\author{
Joveeta Joseph $\mathbb{D}^{1} \cdot$ Bhavani Sontam ${ }^{1} \cdot$ Sai Jeevana Madhuri Guda ${ }^{1}$ Jaishree Gandhi ${ }^{1} \cdot$ Savitri Sharma ${ }^{1}$. \\ Mudit Tyagi $^{2} \cdot$ Vivek Pravin Dave ${ }^{2} \cdot$ Taraprasad Das $\mathbb{D}^{2}$
}

Received: 31 August 2018 / Revised: 22 January 2019 / Accepted: 7 February 2019 / Published online: 21 February 2019

(c) The Royal College of Ophthalmologists 2019

\begin{abstract}
Purpose To determine trends in the microbial spectrum of endophthalmitis over the past 25 years and to review its antibiotic susceptibility patterns over the last 10 years.

Methods Microbiology records of culture-positive endophthalmitis cases from 1991 to 2015 were reviewed. Additionally, data between 2005 and 2015 was also analyzed for trends in antibiotic susceptibility.

Results Of the total of 9278 patients, 3319 (35.7\%) were culture positive and included bacteria $(2840 / 3319,85.56 \%)$, fungi $(387 / 3319,11.66 \%)$, and mixed cultures $(92 / 3319,2.7 \%)$. Gram-positive bacteria accounted for $67.68 \%(1922 / 2840)$ of the total bacteria seen, with the most prevalent pathogen being Streptococcus pneumoniae and Staphylococcus epidermidis. Among the gram-negative organisms Pseudomonas aeruginosa was the most prevalent while. Aspergillus flavus was the most common fungus isolated and Candida sp. accounted for $6.9 \%$ of the total fungi isolated. There was no significant change in the trends of bacteria isolated during the study period. Overall susceptibility patterns showed that gram-positive bacteria were most susceptible to vancomycin $(96 \%)$ and fluoroquinolones $(89 \%)$. The resistance to ceftazidime increased from $31 \%$ in 2005 to $62 \%$ in $2015(P=0.006)$ and amikacin decreased from $36 \%$ in 2005 to $33 \%$ in $2015(P=0.782)$. Although a significant trend $(P<0.001)$ toward increasing microbial resistance against cephalosporins and fluoroquinolones was observed, decreasing microbial resistance against glycopeptides and aminoglycosides was also detected.

Conclusion The spectrum of pathogens causing endophthalmitis at our institute remained similar over the study period. These findings impact the empiric treatment and choice of antibiotics in patients with endophthalmitis.
\end{abstract}

\section{Introduction}

Infectious endophthalmitis remains one of the most challenging complications faced by an ophthalmologist, caused by an array of organisms with devastating outcomes either in the form of permanent blindness or loss of the eyeball itself. Hence, an accurate diagnosis and appropriate and timely treatment are required to prevent this irreversible loss of vision $[1,2]$. Literature reviews show a distinct pattern of variation in the microbial etiology of infectious

Joveeta Joseph

joveeta@1vpei.org

1 Jhaveri Microbiology Centre, Brien Holden Eye Research Centre, L. V. Prasad Eye Institute, Hyderabad, India

2 Smt. Kanuri Santhamma Centre for Vitreo-Retinal Diseases, L. V. Prasad Eye Institute, Hyderabad, India endophthalmitis according to the clinical settings [2-7]. While majority of the reports have been from the USA, there are many reports from other countries that highlight the variation in epidemiology, organism type, their antibiotic susceptibility and treatment outcome in diverse settings [1-5]. Additionally, most single center series describe composite data on either exogenous (post-traumatic, postoperative) or endogenous endophthalmitis (EE), and only two series presents a complete overview of all types of endophthalmitis, over an extended timescale $[8,9]$.

Often empiric intravitreal antibiotics against bacteria (gram-negative and gram-positive pathogens) and/or fungi are the mainstay of treatment 1 , hence, documentation of the trends in causative pathogens of endophthalmitis and their antimicrobial resistance patterns are essential to guide changes in our empiric management of endophthalmitis or reaffirm current practices for prompt management of the disease. The purpose of this study was to determine trends in microorganisms involved in the development of the 
various forms of endophthalmitis presenting in a tertiary eye care referral center in South India for the last 25 years. An attempt was also made to compare the in vitro antibacterial susceptibility pattern of bacterial pathogens recovered from endophthalmitis and investigate possible trends of changes in the susceptibility patterns over the past 10 years.

\section{Methods}

This was a retrospective, non-comparative, consecutive case series. Microbiology records were reviewed of all the culture-proven clinically infectious endophthalmitis cases treated at the L V Prasad Eye Institute, Hyderabad, India, between January 1991 and December 2015. The study was approved by the institutional review board (LEC 0717-060) and adhered to the guidelines of the Declaration of Helsinki. After informed consent vitreous samples from these patients had been investigated for presence of both bacteria and fungus using institutional protocol as described earlier [10]. Briefly, the sample was inoculated directly onto two $5 \%$ sheep blood agar and one each of $5 \%$ sheep blood chocolate agar, Sabouraud dextrose agar, potato dextrose agar, thioglycollate broth, and brain-heart infusion broth. All media were incubated at $37^{\circ} \mathrm{C}$ except Sabouraud dextrose agar and potato dextrose agar, which were incubated at $25-27^{\circ} \mathrm{C}$ for a period of 7 days. Chocolate agar was incubated in the presence of $5 \% \mathrm{CO}_{2}$ and blood agar for anaerobic culture was incubated in anaerobic pouch (Himedia, Mumbai). All other media were incubated aerobically. A culture was considered positive when there was growth of the same organism on two or more media, and/or confluent growth at the site of inoculation on one solid medium, and/or growth in one medium with consistent microscopy findings. In case of positive cultures, the bacteria were further subjected to identification and antibiotic susceptibility testing. The bacterial isolates were identified by conventional biochemical tests and/or API system (bioMérieux, France) and/or Vitek 2 Compact (bioMérieux, France) automated identification systems. Only unequivocal or significant culture results were considered.

Antibiotic susceptibility test was determined by the Kirby-Bauer disk diffusion method. Clinical and Laboratory Standards Institute guidelines (2009) [11] were followed in performing the tests and interpretation. For data analysis of antibiotic susceptibility tests done in last 10 years, analysis for trend was performed using data from consecutive 2-year intervals. Fungal species were identified based on their colony characteristics and microscopic features. Susceptibility for fungal isolates was not performed.

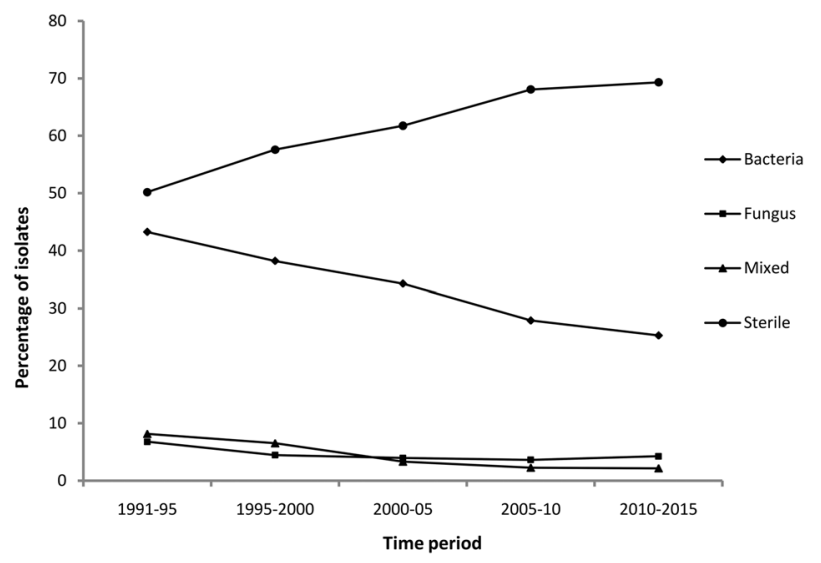

Fig. 1 Trends in endophthalmitis seen at our institute over a 25-year study period from 1991-2015

\section{Results}

Over the 25 years of the study, a total of 9278 patients were clinically diagnosed as infectious endophthalmitis during the study period, of which $3283(35.38 \%)$ were proven culture-positive endophthalmitis. As shown in Fig. 1, the probability of obtaining a vitreous culture-positive for organisms declined with increasing time period and the rate of culture negativity increased over the 25-year study from 51 to $69 \%(p<0.001)$. There was no significant change in number of fungal or mixed endophthalmitis over the study period. Of the 3319 cases with vitreous cultures positive for organisms, fungi were identified in 387 samples $(11.66 \%$ of total cases) and bacteria were identified in 2840 samples ( $85.56 \%$ of the total cases). Additionally, mixed infections were observed in 92 (2.7\%) (Fig. 1). The annual percentage of bacteria isolated among the culture-positive samples, increased over the 25 -year study from $62 \%$ to $85.56 \%(p=$ 0.048 ) and the number of fungal positivity declined from $37.5 \%$ to $11.6 \%(p=0.01)$.

\section{Trends in microbiological spectrum}

A complete picture of 2840 bacterial pathogens isolated from aqueous/vitreous is presented in Table 1, along with the trends in major pathogens causing endophthalmitis (Fig. 2). Gram-positive cocci accounted for 1477 (52\%) of all bacterial isolates, gram-positive bacilli for 445 (15.7\%), gram-negative cocci for 81 (2.8\%), gram-negative bacilli for $837(29.5 \%)$, and acid-fast or partially acid-fast organisms for $36(1.3 \%)$ of all isolates. Overall among the total bacteria, $67.7 \%$ of isolates were gram-positive, $32.3 \%$ were gram-negative. The most common pathogens were Streptococcus pneumoniae (15.4\%), followed by Staphylococcus epidermidis (14.8\%), Alpha-hemolytic streptococci (4.75\%), and Bacillus spp. (4.3\%). Among the gramnegative organisms isolated, Pseudomonas aeruginosa 
Table 1 Endophthalmitis bacterial isolates from 1991 to 2015

\begin{tabular}{|c|c|c|c|}
\hline S1. no & Isolates & Number & $\%$ Total \\
\hline $\mathbf{A}$ & Gram-positve bacteria & 1922 & 67.68 \\
\hline 1 & Staphylococcus epidermidis & 420 & 14.79 \\
\hline 2 & Staphylococcus aureus & 77 & 2.71 \\
\hline 3 & Coagulase negative Staphylococcus & 56 & 1.97 \\
\hline 4 & Staphylococcus hominis & 23 & 0.81 \\
\hline 5 & Staphylococcus hemolyticus & 21 & 0.74 \\
\hline 6 & Streptococcus pneumoniae & 437 & 15.39 \\
\hline 7 & Alpha hemolytic Streptococci & 135 & 4.75 \\
\hline 8 & Streptococcus pyogenes & 36 & 1.27 \\
\hline 9 & Streptococcus mitis & 27 & 0.95 \\
\hline 10 & Other Bacillus species & 122 & 4.3 \\
\hline 11 & Bacillus cereus & 39 & 1.37 \\
\hline 12 & Bacillus licheniformis & 38 & 1.34 \\
\hline 13 & Bacillus coagulans & 29 & 1.02 \\
\hline 14 & Corynebacterium species & 34 & 1.2 \\
\hline 15 & Nocardia species & 17 & 0.6 \\
\hline 16 & Propionibacterium acnes & 5 & 0.18 \\
\hline B & Gram negative bacteria & 918 & 32.32 \\
\hline 1 & Haemophilus influenzae & 34 & 1.2 \\
\hline 2 & Pseudomonas aeruginosa & 259 & 9.12 \\
\hline 3 & Burkholderia cepacia & 38 & 1.34 \\
\hline 4 & Escherichia coli & 59 & 2.08 \\
\hline 5 & Enterobacter species & 54 & 1.9 \\
\hline 6 & Klebsiella pneumoniae & 21 & 0.74 \\
\hline 7 & Proteus mirabilis & 5 & 0.18 \\
\hline
\end{tabular}

Bold values are overall percetages for that group of organisms

(9.12\%), E.coli $(2.08 \%)$ were encountered most frequently (Table 1).

As an exploratory analysis, we assessed for trends in the most common organisms causing infectious endophthalmitis over time and the trends in yearly incidence of these major gram-positive and gram-negative pathogens are shown in Fig. 2a, b. The spectrum of organisms seen at our institute remained the same over the 25-year period with Pseudomonas aeruginosa being the most common gramnegative pathogen isolated, followed by other Enterobacteriaceae organisms. Among the gram-positive organisms isolated, there was decreasing trend in coagulase negative staphylococci (42\% in 1991 to $29 \%$ in 2015) and increasing trend in isolation of Streptococcus sp. (30\% in 1991 to $39 \%$ in 2015), though not statistically significant.

The percentages of Bacillus and Corynebacterium spp. remained constant over the 25-year period. Among the fungal species isolated during the study period, Aspergillus flavus $(66 / 387,17.05 \%)$ and Fusarium sp. (43/387, 11\%) was the most frequently isolated fungus followed by unidentified hyaline and dematiaceous fungi (Table 2). An
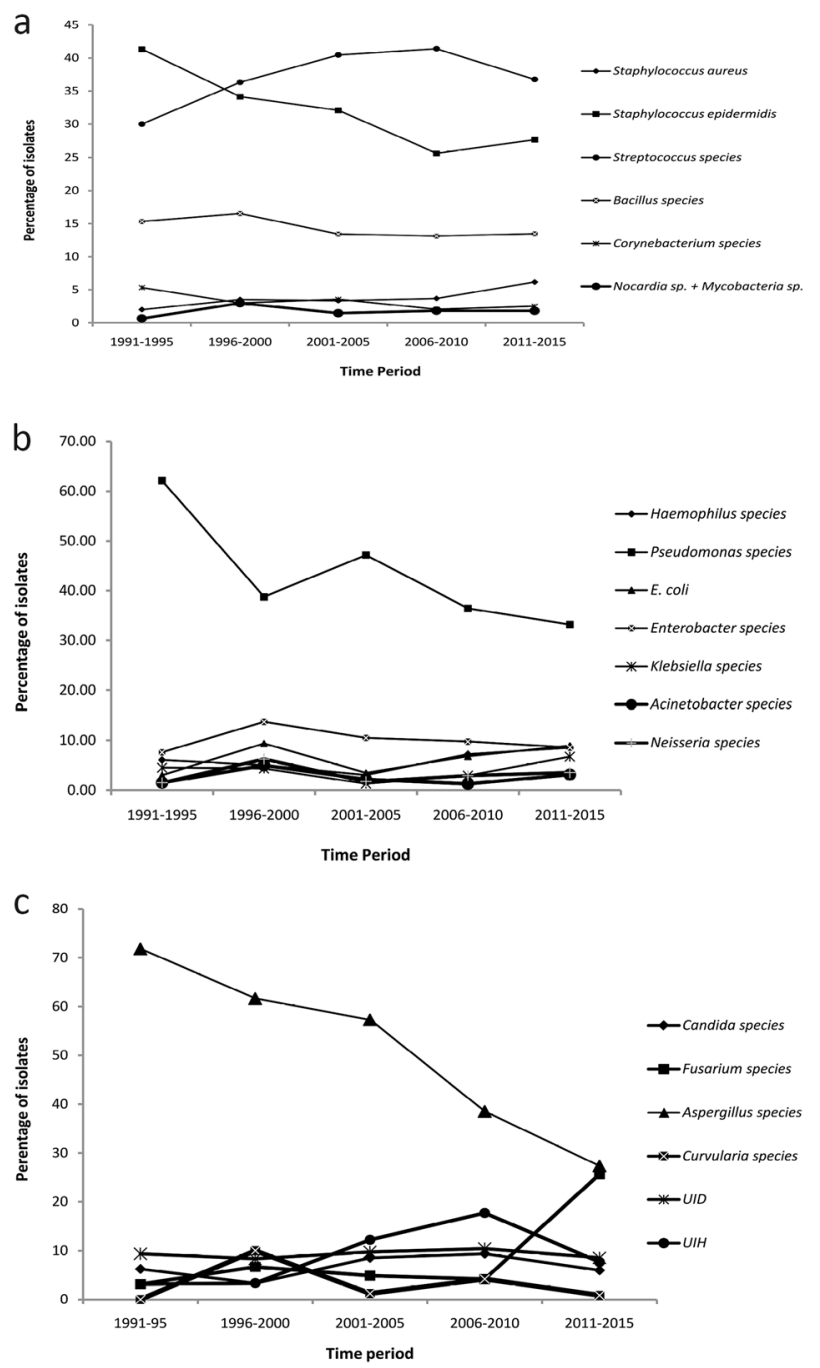

Fig. 2 Trends in prevalence of major pathogens of (a) gram positive and (b) gram negative bacteria causing infectious endophthalmitis during the 25-year study period. $\mathbf{c}$ Trends in incidence of major fungal pathogens causing infectious endophthalmitis during the study period from 1991-2015

interesting trend observed was the decrease in Aspergillus sp. isolated, from $72 \%$ in $1991-1995$ to $27.3 \%(p<0.0001)$ in 2015 and an increase in Fusarium sp. isolated from 3.1\% in 1995 to $25.6 \%(p<0.005)$ in 2016 as shown in Fig. 2c.

\section{Trends in antibiotic susceptibilities}

When the trends in susceptibility to various ocular antibiotics were observed, there was decrease in susceptibility to almost all antibiotics studied over the 10-year period. Susceptibility profiles of gram-positive and gram-negative bacterial pathogens to selected ocular antibiotics are illustrated in Fig. 3. Overall susceptibility pattern in our study showed that gram-positive bacteria were most susceptible to glycopeptides like vancomycin (96\%) and fluoroquinolones 
Table 2 Type of fungi isolated from patients with endophthalmitis from 1991 through 2015

\begin{tabular}{|c|c|c|}
\hline Type of fungal isolates & Number & $\%$ \\
\hline Filamentous fungus & 360 & 93.02 \\
\hline Hyaline fungi & 275 & 71.06 \\
\hline Aspergillus flavus & 66 & 17.05 \\
\hline Aspergillus niger & 24 & 6.20 \\
\hline Aspergillus terreus & 35 & 9.04 \\
\hline Aspergillus fumigatus & 35 & 9.04 \\
\hline Aspergillus nidulans & 1 & 0.26 \\
\hline Aspergillus species & 15 & 3.88 \\
\hline Fusarium species & 37 & 9.56 \\
\hline Fusarium solani & 5 & 1.29 \\
\hline Fusarium moniliforme & 1 & 0.26 \\
\hline Penicillium species & 3 & 0.78 \\
\hline Pencillium marneffei & 2 & 0.52 \\
\hline Paecilomyces species & 1 & 0.26 \\
\hline Acremonium species & 7 & 1.81 \\
\hline Apophysomyces elegans & 1 & 0.26 \\
\hline Chrysosporium species & 3 & 0.78 \\
\hline Unidentified hyaline fungus & 39 & 10.08 \\
\hline Dematiaceous fungi & 85 & 21.96 \\
\hline Curvularia species & 6 & 2.55 \\
\hline Aureobasidium pullulans & 1 & 0.26 \\
\hline Chaetomium species & 1 & 0.26 \\
\hline Cladosporium species & 6 & 1.55 \\
\hline Colletotrichum species & 2 & 0.52 \\
\hline Colletotrichum coccodes & 1 & 0.26 \\
\hline Colletotrichum dematium & 2 & 0.52 \\
\hline Unidentifed dematiaceous fungus & 42 & 10.85 \\
\hline Yeasts & 27 & 6.98 \\
\hline Candida albicans & 14 & 3.62 \\
\hline Candida species & 13 & 3.36 \\
\hline
\end{tabular}

Bold values are overall percetages for that group of organisms

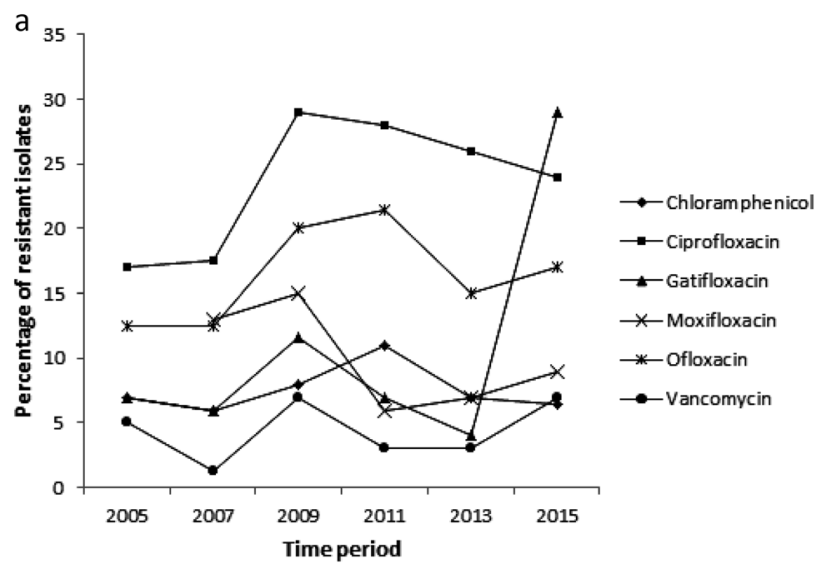

like gatifloxacin $(89 \%)$ and moxifloxacin $(89.9 \%)$. The susceptibility pattern of gram-negative organisms towards fluoroquinolones ranged between $67-79 \%$. The resistance to ceftazidime increased from $31 \%$ in 2005 to $62 \%$ in 2015 $(P=0.0073)$ and amikacin decreased from $36 \%$ in 2005 to $33 \%$ in $2015(P=0.78)$.

\section{Discussion}

A variety of factors determine the incidence of endophthalmitis and spectrum of organisms vary from one country to the other and in different geographical areas in the same country. The endophthalmitis data generated from our institute in South India is more than a decade old [12, 13]. Since environmental conditions, surgical techniques, and aseptic practices have evolved, there is a possibility of a change in the microbiology and antibiotic sensitivity patterns of endophthalmitis with time, and thus, there is a need for more recent report over an extended time period. In our institute, however, there was no major change in the microbiologic spectrum in a 25 -year period. The majority of endophthalmitis seen in our country is bacterial and mainly involves Streptococcus sp. and Staphylococcus sp, which is consistent with most published studies [3, 4, 7, 8]. However, there is a very significant presence of gram-negative and fungal organisms in our series as is the case with studies from this part of the world [8]. While there is an increase in the number of Streptococcus sp. causing endophthalmitis over the 25-year period, a reduction in the Staphylococcus epidermidis was noticed possibly due to the identification of newer organisms in ViTEK 2, which was earlier identified as coagulase-negative staphylococci. Among gram-negative bacteria, however $P$. aeruginosa continues to be the most common pathogen over 25 years at our institute. Fungal organisms particularly the filamentous pathogens like Aspergillus sp. and Fusarium sp. make upto 5-10\% of the



Fig. 3 Trends of antibiotic resistance in (a) gram-positive organisms and (b) gram-negative organisms from 2005-2015 
total microbes causing endophthalmitis. Candida sp. endophthalmitis also has been reported and this trend has been increasing lately especially in cases of Descemet stripping automated endothelial keratoplasty (DSAEK) which has been reported to be associated with Candida parapsilosis [14] or where there is a history of longer donor cornea storage times and the spread is from the donor tissue. An important outcome of this study was observation of increase in the rate of culture negativity from 51 to $69 \%$ over the 25-year period. While the etiologic basis of culturenegative endophthalmitis remain unclear, possibilities for this condition include, presence of fastidious organisms and prior antimicrobial therapy that may inhibit microbial growth during culture or infection with unculturable pathogens to scant (undetectable) bacterial pathogens. Newer diagnostic techniques using molecular methods may provide more accurate and more sensitive results than traditional culture methods, although at the present time these techniques are not widely available outside of major medical centers.

Empiric treatment of endophthalmitis with broadspectrum antibiotics based on the susceptibility patterns in that country is very important for good anatomical and visual outcomes. Consistent with the ability of bacteria to adapt to changing selective pressures, isolates from patients with endophthalmitis have also shown a significant trend toward both increasing resistance to ceftazidime and fluoroquinolones and decreasing resistance to aminoglycosides and imipenem. Drug resistance is an emerging problem especially among Gram-negative isolates causing acuteonset post-operative endophthalmitis in India. This has important implications for the empiric use of antibiotics against these resistant strains, and also in the increasing spread of this resistance in the population [15]. Regional variability in susceptibilities of bacteria to selected antibiotics should serve as a warning to all to analyze trends in local antibiotic susceptibility and resistance patterns on a regular basis in order to achieve successful outcomes. Limitations of our study include its retrospective nature wherein the diagnosis of endophthalmitis was available in the microbiology records, hence, clinical data could not be added, which has limited our ability to perform additional analyses, including visual outcomes and subgroup analysis of endophthalmitis types. Additionally, we have earlier published that in all types of endophthalmitis (post-operative/traumatic or endogenous) the spectrum of organisms is similar [16] and empirical use of antibiotics is based on the microbiological evidence rather than the pre-disposing factor. While clinical correlation would have provided more robust information to our study, retrieving the clinical information in this large data set was difficult.

In conclusion, our study found that there was no significant change in the spectrum of micro organisms over the past 25 years at the L V Prasad Eye Institute, India, though it differs from the western literature in having a high incidence of gram-negative and fungal etiology causing endophthalmitis. Vancomycin continues to be an excellent empiric antibiotic for treating endophthalmitis caused by gram-positive organisms, however for gram-negative organisms, drugs like colistin or imipenem may be considered as an alternative in the management of these cases.

\section{Summary}

\section{What was known before}

- Gram-positive bacteria is the most predominant ethology in endophthalmitis. Ceftazidime and Vancomycin are most commonly used in the treatment of endophthalmitis.

\section{What this study adds}

- The microbial spectrum of endophthalmitis remained similar over 25 years in India and included bacteria $(85.56 \%)$ and fungi $(11.66 \%)$. Vancomycin is an excellent empiric antibiotic for treating endophthalmitis, while increasing resistance to ceftazidime is a concern.

Acknowledgements We thank The Hyderabad Eye Research Foundation, Hyderabad.

Funding This work was supported by a grant from the DST-SERB to Dr Joveeta Joseph (File Number: EMR/2016/002259) India.

\section{Compliance with ethical standards}

Conflict of interest The authors declare that they have no conflict of interest.

Publisher's note: Springer Nature remains neutral with regard to jurisdictional claims in published maps and institutional affiliations.

\section{References}

1. Durand ML. Endophthalmitis. Clin Microbiol Infect. 2017;19:227-34.

2. Callegan MC, Engelbert M, Parke DW II, Jett BD, Gilmore MS. Bacterial endophthalmitis: epidemiology, therapeutics, and bacterium-host interactions. Clin Microbiol Rev. 2002;15:111-24.

3. Han DP, Wisniewski SR, Wilson LA, Barza M, Vine AK, Doft $\mathrm{BH}$, et al. Spectrum and susceptibilities of microbiologic isolates in the Endophthalmitis Vitrectomy Study. Am J Ophthalmol. 1996;122:1-17.

4. Jackson TL, Eykyn SJ, Graham EM, Standford MR. Endogenous bacterial endophthalmitis: a 17 year prospective series and review of 267 reported cases. Surv Ophthalmol. 2003;48:403-24. 
5. Das TP, Kunimoto DY, Sharma S, Jalali S, Majji AB, Rao TN, et al. Relationship between clinical presentation and visual outcome in postoperative and posttraumatic endophthalmitis in south central India. Indian J Ophthalmol. 2005;53:5-16.

6. Scott IU, Flynn HW, Feuer W, Pflugfelder SC, Alfonsa EC, Forster RK, et al. Endophthalmitis associated with microbial keratitis. Ophthalmology. 1996;103:1864-70.

7. Satpathy G, Nayak N, Wadhwani M, Venkwatesh P, Kumar A, Sharma Y, et al. Clinicomicrobiological profile of endophthalmitis: a 10 year experience in a Tertiary Care Center in North India. Indian J Pathol Microbiol. 2017;60:214-20.

8. Gentile RC, Shukla S, Shah M, et al. Microbiological spectrum and antibiotic sensitivity in endophthalmitis: a 25-year review. Ophthalmology. 2014;121:1634-42.

9. Sharma S, Padhi TR, Basu S, Kar S, Roy A, Das TP. Endophthalmitis patients seen in a tertiary eye care centr e in Odisha: A clinic-microbiological analysis. Indian J Med Res. 2014;139:91-8.

10. Sharma S, Jalali S, Adiraju MV, Gopinathan U, Das T. Sensitivity and predictability of vitreous cytology, biopsy, and membrane filter culture in endophthalmitis. Retina. 1996;16:525-9.

11. Lalitha P, Sengupta S, Ravindran RD, Sharma S, Joseph J, Ambiya $\mathrm{V}$, et al. A literature review and update on the incidence and microbiology spectrum of post-cataract surgery endophthalmitis over past 2 decades in India. Indian $\mathbf{J}$ Ophthalmol. 2017;65:673-7.

12. Kunimoto DY, Das T, Sharma S, Jalali S, Majji A, Gopinathan U, et al. Microbiologic spectrum and susceptibility of isolates. Part I. Postoperative endophthalmitis. Am J Ophthalmol. 1999;128: 240-2.

13. Kunimoto DY, Das T, Sharma S, Jalali S, Majji A, Gopinathan U, et al. Microbiologic spectrum and susceptibility of isolates. Part II. Posttraumatic endophthalmitis. Am J Ophthalmol. 1999;128: 242-4.

14. Chew AC, Mehta JS, Li L, Busmanis I, Tan DT. Fungal endophthalmitis after descemet stripping automated endothelial keratoplasty-A case report. Cornea. 2010;29:346-9.

15. Pathengay A, Moreker MR, Puthussery R, Ambatipudi S, Jalali S, Majji $\mathrm{AB}$, et al. Clinical and microbiologic review of culture proven endophthalmitis caused by multidrug resistant bacteria in patients seen at a tertiary eye care centre in southern India. Retina. 2011;31:1806-11.

16. Sharma S, Padhi TR, Basu S, Kar S, Roy A, Das T. Endophthalmitis patients seen in a tertiary eye care centre in Odisha: a clinicomicrobiological analysis. Indian J Med Res. 2014;139:91-8. 